\title{
Editorial
}

\section{Coupled Numerical Methods in Engineering Analysis}

\author{
Delfim Soares Jr., ${ }^{1}$ Otto von Estorff, ${ }^{2}$ \\ Jan Sladek, ${ }^{3}$ and Luis Godinho ${ }^{4}$ \\ ${ }^{1}$ Structural Engineering Department, Federal University of Juiz de Fora, Juiz de Fora, \\ MG 36036-330, Brazil \\ ${ }^{2}$ Institute of Modeling and Computation, Hamburg University of Technology, \\ 21073 Hamburg, Germany \\ ${ }^{3}$ Department of Mechanics, Slovak Academy of Sciences, 84503 Bratislava, Slovakia \\ ${ }^{4}$ CICC - Research Center in Construction Sciences, Department of Civil Engineering, \\ University of Coimbra, 3030-788 Coimbra, Portugal
}

Correspondence should be addressed to Delfim Soares Jr., delfim.soares@ufjf.edu.br

Received 11 December 2011; Accepted 12 December 2011

Copyright (C) 2011 Delfim Soares Jr. et al. This is an open access article distributed under the Creative Commons Attribution License, which permits unrestricted use, distribution, and reproduction in any medium, provided the original work is properly cited.

Complex engineering analyses usually require the use of numerical methods to provide accurate data. Although nowadays there are several powerful numerical techniques available, such as the Finite Element Method, the Finite Volume Method, the Boundary Element Method, and Meshless Methods, none of them can be considered most appropriate for all kinds of analysis, and usually the coupling of different numerical methodologies is necessary to analyze complex problems more effectively. In this context, several coupling procedures have been proposed over the last decades considering different numerical methods, in order to profit from their respective advantages. The papers selected for this special issue represent a good panel for addressing this challenging theme. Although different kinds of coupling procedures and hybrid formulations are presented here to analyze multiphysics behavior, of course, they are not an exhaustive representation of the vast area of coupled numerical methods in engineering analysis. Nonetheless, they represent the rich and many-faceted knowledge, that we have the pleasure of sharing with the readers. Therefore, we would like to thank the authors for their contributions and the reviewers for all their fundamental work on these papers.

This special issue contains twelve papers, which are organized considering firstly interface coupled analyses and, later on, domain coupled models. Considering interface 
coupling procedures, initially, papers discussing fluid-solid coupled systems are presented, being, in the sequence, papers based on solid-solid coupled models enumerated. Regarding domain coupled analyses, the papers presented here are mostly related to computational fluid models. It is important to remark that several coupled numerical procedures, taking into account a vast game of numerical techniques, are discussed in this issue, highlighting the richness and variety of the theme.

In the paper entitled "Coupled numerical methods to analyze interacting acoustic-dynamic models by multidomain decomposition techniques," several numerical methods, such as the Finite Difference Method, the Finite Element Method, the Boundary Element Method, and Meshless Methods, are considered to model each subdomain of the fluid-solid coupled model, and multidomain decomposition techniques are applied to deal with the coupling relations. In this case, completely independent spatial and temporal discretizations among the interacting subdomains are permitted and coupling algorithms based on explicit and implicit timemarching techniques are discussed.

In the paper entitled "Finite element analysis of dam-reservoir interaction using high-order doubly asymptotic open boundary," direct and partitioned coupled methods are developed to analyze a dam-reservoir system, which is divided into the near-field, modeled by the finite element method, and the far-field, modeled by the high-order doubly asymptotic open boundary (DAOB). In the direct coupled method, a symmetric monolithic governing equation is formulated by incorporating the DAOB with the finite element equation, which is solved using standard time-integration methods. In the partitioned coupled method, the near-field finite element equation and the far-field DAOB condition are separately solved and coupling is achieved by applying the interaction force on the truncated boundary, taking into account an iteration strategy, which is employed to improve the numerical stability and accuracy of the methodology.

In the paper entitled "Coupling the BEM/TBEM and the MFS for the numerical simulation of wave propagation in heterogeneous fluid-solid media," wave propagation in an elastic medium containing elastic, fluid, rigid and empty heterogeneities is simulated, taking into account frequency-domain analyses. In this case, a coupling formulation between the boundary element method (BEM)/the traction boundary element method (TBEM) and the method of fundamental solutions (MFS) is employed. Thus, the full domain is divided into subdomains, which are handled separately by the BEM/TBEM or the MFS, being the coupling enforced by applying the prescribed boundary conditions at all medium interfaces.

In the paper entitled "A hybrid analytical-numerical model based on the method of fundamental solutions for the analysis of sound scattering by buried shell structures," a hybrid numerical-analytical model is proposed to address the problem of underwater sound scattering by an elastic circular shell structure that is buried in a fluid seabed, below a water waveguide. In this case, the coupling between the analytical solutions developed both for sound propagation in the waveguide and in the vicinity of the circular hollow shell is performed using the Method of Fundamental Solutions; the proposed strategy allows a compact description of the propagation medium and claims to be accurate and efficient from the computational point of view.

In the paper entitled "Dynamic analysis of partially embedded structures considering soilstructure interaction in time domain," the substructure method, using the dynamic stiffness of soil, is used to analyze soil-structure systems. In this case, a time-domain coupled model based on the finite element method and on the scaled boundary finite element method is applied, where the finite element method is used to analyze the structure and the scaled boundary finite element method is applied in the analysis of the unbounded soil region. 
In the paper entitled "Stress analysis of three-dimensional media containing localized zone by FEM-SGBEM coupling," an efficient numerical technique for three-dimensional stress analysis of an infinite medium containing a line of singularity introduced by dislocations and cracks and a localized region exhibiting complex behaviors (e.g., material nonlinearities and material inhomogeneities) is proposed. In this case, a coupling procedure exploiting positive features of both standard finite element method (FEM) and symmetric Galerkin boundary element method (SGBEM) is considered. Thus, an infinite medium is decomposed into two subdomains such that a finite subdomain that is of small size and may contain a region exhibiting complex behavior is modeled by the FEM while the other (compliment) unbounded subdomain that may contain cracks is treated by the weakly singular SGBEM.

In the paper entitled "A corotational finite element method combined with floating frame method for large steady-state deformation and free vibration analysis of a rotating-sinclined beam," large steady-state deformation and infinitesimal-free vibration around the steady state deformation of a rotating-inclined Euler beam at constant angular velocity are analyzed. In this analysis, the authors take into account the corotational finite element method combined with the floating frame method.

In the paper entitled "Interior noise prediction of the automobile based on hybrid FE-SEA method," a hybrid FE-SEA method (where FE stands for Finite Element and SEA stands for Statistical Energy Analysis) is employed to predict the interior noise of the automobile in the low- and middle-frequency band in the design and development stage. This is based on the prediction of parameters, which include modal density, damping loss factor and coupling loss factor, and excitations, which incorporate sound excitation of engine cabin, excitation of engine mounts, excitation of road roughness, and wind excitations.

In the paper entitled "A new reduced stabilized mixed finite-element method based on proper orthogonal decomposition for the transient Navier-Stokes equation," a reduced stabilized mixed finite element (RSMFE) formulation based on proper orthogonal decomposition (POD) for the transient Navier Stokes equations is presented. In this case, by combining the POD with the SMFE formulation, a new low dimensional and highly accurate SMFE method for the transient Navier Stokes equations is obtained, which not only reduces the degrees of freedom but also circumvents the constraint of inf-sup stability condition.

In the paper entitled "A fully discrete Galerkin method for a nonlinear space-fractional diffusion equation," a fully discrete scheme for a type of nonlinear space-fractional anomalous advection-diffusion equation is presented, where, in the spatial direction, the finite element method is used, whereas, in the temporal direction, the modified Crank-Nicolson approximation is employed.

In the paper entitled "A numerical treatment of nondimensional form of water quality model in a nonuniform flow stream using Saulyev scheme," mathematical models are used to simulate pollution due to sewage effluent and a finite difference scheme for solving the advectiondispersion-reaction equations (ADRE) of the uniform flow model is considered, as well as the effect of nonuniform water flows in a stream.

Finally, in the paper entitled "Modeling and simulation of fiber orientation in injection molding of polymer composites," the fundamental modeling and numerical simulation for a prediction of fiber orientation during injection molding process of polymer composite is reviewed. In general, the simulation of fiber orientation involves coupled analysis of flow, temperature, moving-free surface, and fiber kinematics. Therefore, coupled numerical methods are needed to analyze these complex problems. Thus, in this last paper, several well-established methods, such as the finite-element/finite-different hybrid scheme for the Hele-Shaw 
flow model and the finite element method for the general three-dimensional flow model, are reviewed.

Delfim Soares Jr.

Otto von Estorff

Jan Sladek

Luis Godinho 


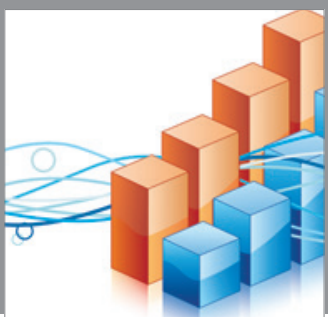

Advances in

Operations Research

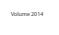

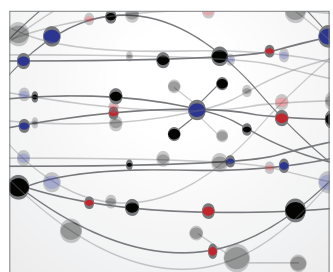

\section{The Scientific} World Journal
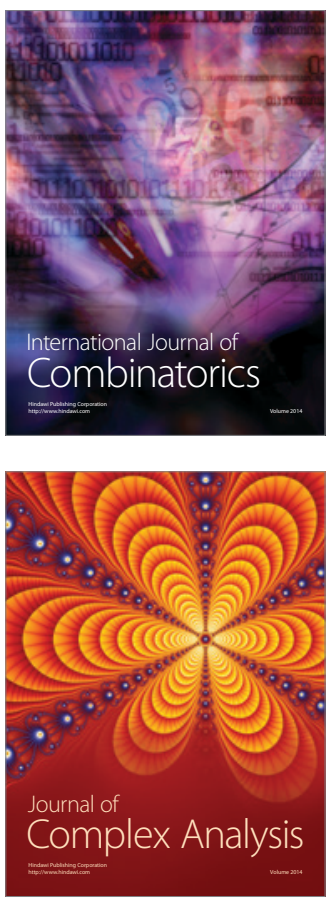

International Journal of

Mathematics and

Mathematical

Sciences
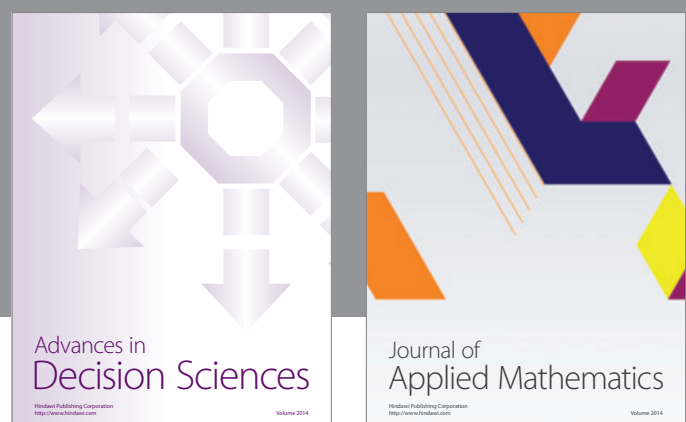

Journal of

Applied Mathematics
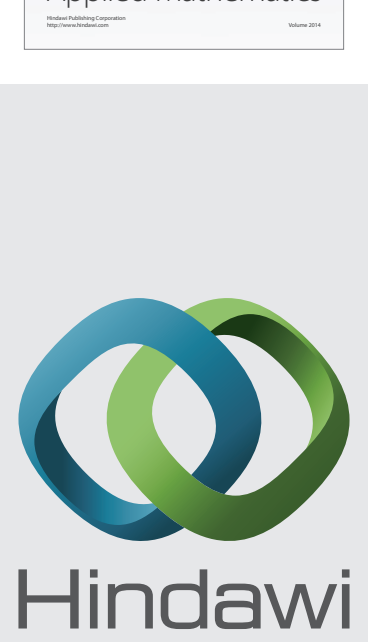

Submit your manuscripts at http://www.hindawi.com
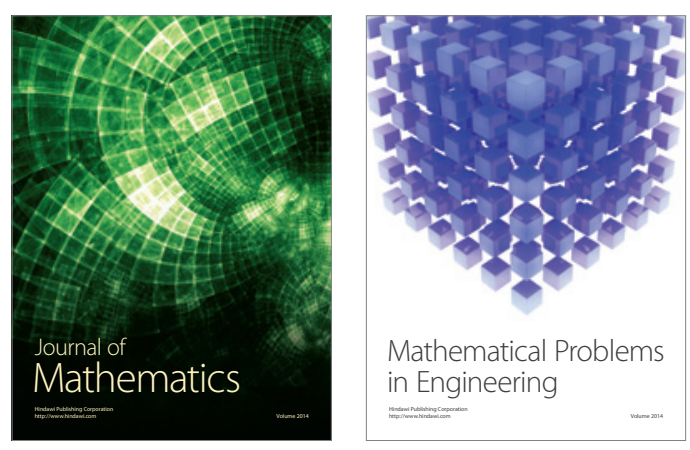

Mathematical Problems in Engineering
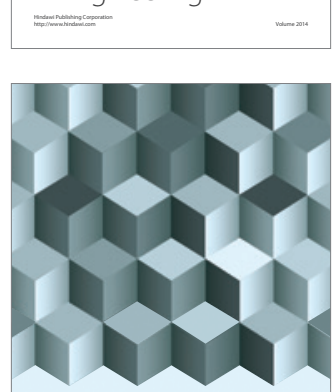

Journal of

Function Spaces
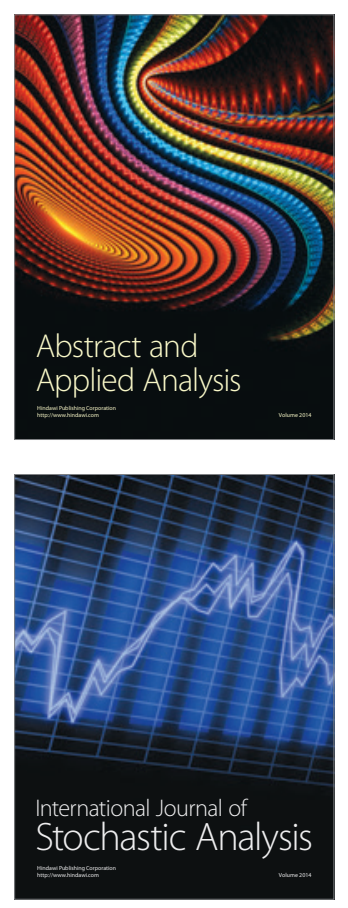

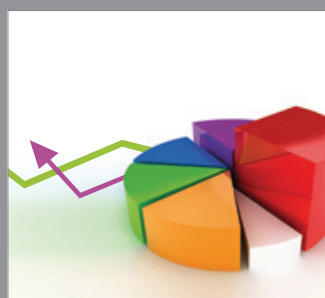

ournal of

Probability and Statistics

Promensencen
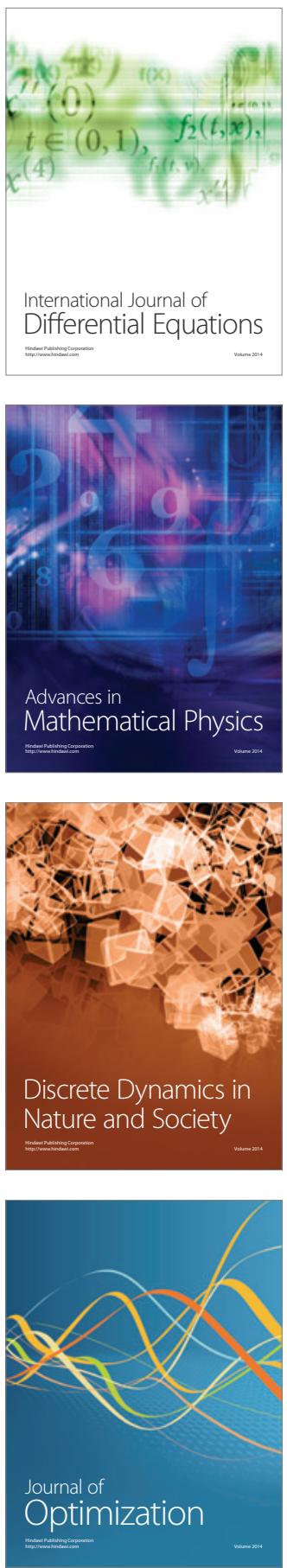